\title{
Waypoint Planning for Autonomous Aerial Inspection of Large-Scale Solar Farms
}

\author{
Ehab Salahat, Charles-Alexis Asselineau, Joe Coventry and Robert Mahony \\ Research School of Electrical, Energy and Materials Engineering, Australian National University, ACT, Australia
}

\begin{abstract}
Solar energy is seen as a sustainable and nondepletable source of energy supply. Worldwide, large-scale solar power infrastructure is being installed every day. Such structures can suffer from many faults and defects that degrade their energy output during their operational life. Detecting such faults and defects requires regular inspection over physically large and distributed solar infrastructure. On-site manual human inspection tends to be impractical, risky and costly. As such, replacing humans with autonomous robotic aerial inspection systems has great potential. In this work, we propose an unmanned aerial vehicle (UAV) waypoint generation system that is specifically designed for aerial inspection of solar infrastructure. Our system takes into consideration the physical structure and the dynamic nature of sun-tracking solar modules and generates waypoints with the right camera viewing pose and drone orientation. Statistical methods are used to generate a randomly selected set of modules as a representation of the entire solar farm. The set is guaranteed to satisfy a user-defined confidence level and margin of error requirements. A path is generated to visit selected modules in an optimal way by deploying the traveling-salesman shortest path algorithm, allowing the vehicle to maximize battery use. Illustrative flights and preliminary inspection results are presented and discussed.
\end{abstract}

\section{INTRODUCTION}

Solar energy is a sustainable alternative source of energy to fossil fuels and has the potential to make a major contribution to the world's energy needs [1]. In fact, the world's overall solar energy potential is around 5.6 gigajoules (GJ) (1.6 megawatt-hours (MWh)) per square meter per year, which far exceeds total world energy demand [2]. For example, the annual solar radiation falling on Australia is approximately 58 million petajoules (PJ), approximately 10000 times Australia's annual energy consumption of 5772 PJ in 2007 [2]. Theoretically, if only $0.1 \%$ of the incoming radiation is converted into usable energy at an efficiency of $10 \%$, all Australia's energy needs could be supplied by solar energy [2].

The solar energy market is booming [3] and the cost of the technology is decreasing. Large-scale deployment of solar power facilities, especially in the 66 sunbelt countries, is underway, and the rate of deployment will only increase. Fig. 1 illustrates both a photovoltaic (PV) farm and a concentrating solar power (CSP) facility. While both PV and CSP plants are designed to operate for 20 30 years, field observations show that systemic environmental factors such as soiling can significantly degrade their performance. In addition, individual PV modules may develop faults and defects. The identification of such issues requires regular inspection over physically large and distributed solar infrastructure $(>10,000$ modules).

For PV plants, the two common methods for inspection are systemic performance testing and direct visual inspection [4]. Performance testing monitors the electrical characteristics of the module $[5,6]$. Visual inspection is a non-invasive

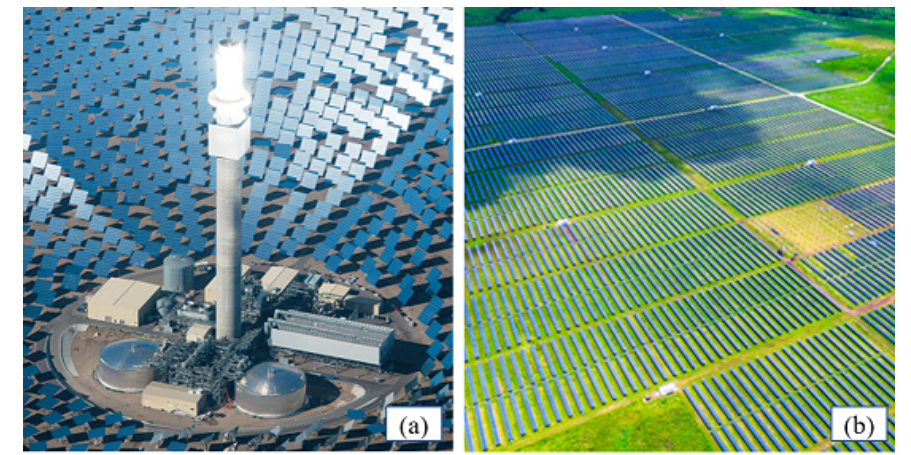

Fig. 1: (a) Solar thermal and (b) solar photovoltaic installations.

and non-destructive method that uses visible, infrared and hyper-spectral camera images to infer information about the performance of the module. Visual inspection is particularly well suited to the identification of physical damage and soiling. Presently, on-site visual inspection is performed manually by maintenance personnel on a regular basis [7], see Fig. 2. Manual visual inspection suffers from being highly subjective and results are difficult to reproduce [8]. Moreover, solar power plants are often operated in inhospitable environments (e.g. deserts), and manual inspection requires high safety standards making it slow and expensive, sometimes requiring a temporary shutdown of the facility. The development of an autonomous robotic visual inspection system for solar infrastructure offers the potential to provide up-to-date and detailed information on the operational status of PV modules, allowing operators to maximize value in the operation and maintenance of the facility [9].

Due to their increased availability, cost-effectiveness, and maneuverability, UAVs are a natural candidate for robotic visual inspection of large-scale infrastructure [9]. Their agility and ability to autonomously acquire and accurately register sensor data from multiple sensing modalities (visible light, thermal, near infra-red, hyperspectral, etc) is a key technical capability that promotes them to be deployed for effective long-term operation of renewable energy infrastructure.

For large-scale facilities, an inspection of each and every panel on a regular basis may be infeasible. The size of the facility precludes the drone visiting all modules during every inspection and some sampling strategy will be required. Furthermore due to the changing time of day and season that a module is visited, between each flight, the waypoints will change depending on the angle of the sun and the angle of the modules. Thus, the path followed to visit the selected modules will change for each flight and an automatic path planning algorithms is crucial. Path planning is a well studied question in robotics literature [10] and include randomly sample search 

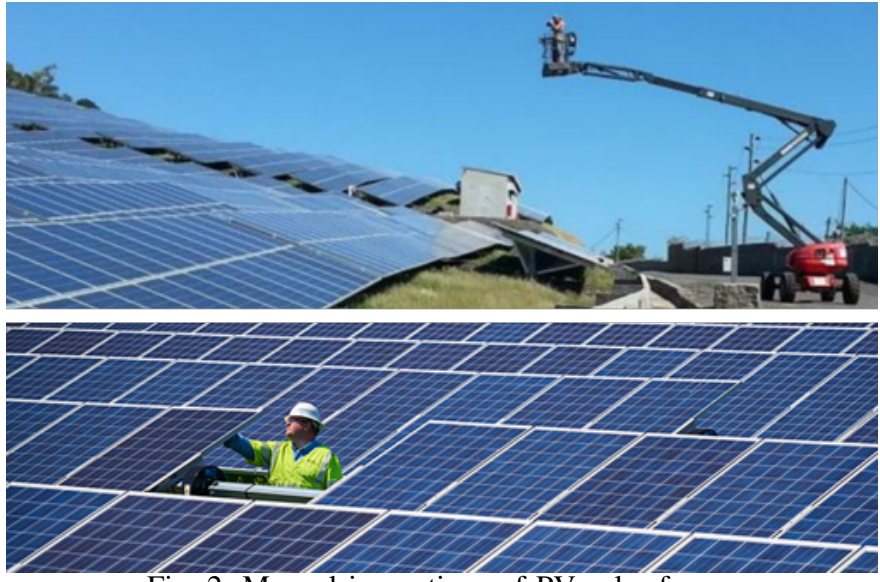

Fig. 2: Manual inspections of PV solar farms.

algorithms such as particle swarm algorithms [11] and Dijsktraâs algorithm [12]. These algorithms are concerned with finding the shortest path that leads to a given destination from a known starting point. However, our problem is fundamentally different. The nodes (modules) can be visited in any order but have a fixed starting and ending point (the take-off point) and a travel distance that should be minimized. To our knowledge, [11] is the only closely related work. There, the authors used particle swarm optimization (PSO) to plan a similar mission using Bezier curves to generate smooth 2D paths. The solar inspection application considered in the present work requires $3 \mathrm{D}$ paths and precise positioning for the inspection photos.

In this paper, we propose a reliable and cost-effective method to inspect a portfolio of solar modules in a large solar facility. We take into account key practical parameters during mission planning, including the changing orientation of the solar modules. We assume that there is an image processing algorithm available that can take the images generated by the inspection process and provide a measure of performance of the panel with some uncertainty $\epsilon$. We propose a statistical sampling algorithm to identify a small subset of the total solar modules to inspect that yields an estimate of performance for the full solar field based on the inspected sample for a given confidence level. We propose using a "travelling salesman algorithm" to generate a minimum distance path that will then visit all modules in the sample set in minimum time. We allow the engineer to optimize between flight time (a function of battery capacity) and inspection confidence by quickly generating multiple sample strategies and associated paths. The proposed suite of algorithms aims to provide highly accurate inspection results with the lowest cost.

The remainder of this paper is structured as follows. In section II, we highlight the importance of waypoint planning for aerial inspection of solar farms. Our system model is introduced in section III-A. Section IV discusses some conducted experiments and real applications. The paper findings and contributions are summarized in section $\mathrm{V}$.

\section{PRELIMINARIES}

\section{A. Surveying vs. Inspection}

One should not confuse visual inspection with visual surveying. Unlike surveying which is typically done from a high altitude ( $\sim 50$ meters), inspection is a more demanding process and requires a higher level of data resolution. It requires an indepth analysis of solar modules which consequently enforces the need for a closer view ( $\sim 5$ meters) and more sophisticated and high resolution imaging as well as accurate positioning equipment to acquire the images. For example, with visual surveying, small defects and other performance degradation mechanisms (e.g., corrosion spots on heliostats) may be at millimeter scale, and need at least a few pixels to resolve. Closer and more detailed sensing by visual inspection has much higher chances of detecting such faults. This argument is also supported by field observation as well as the research work in $[13,14]$ that studies the correlation between the flight height and the ability of fault detection.

\section{B. Waypoint Planning vs. Path Finding}

Though very similar, waypoint planning is different from path finding. For path finding, the 3D structure of travel in a potentially dynamic environment is unknown, and routing decisions are made one step at a time. Path (or waypoint) planning involves generation of a real-time path to the destination, while avoiding obstacles and optimizing a given cost function with kinodynamic constraints [15]. Path finding is better for unknown environments, whereas path planning is more suited to known environments.

In solar farms, where the GPS coordinates of each module and man-made structure are known prior, waypoints can be actually pre-planned before the UAV takes off. The challenge here, however, is that at every waypoint, both the drone and the equipped camera have to be correctly positioned in order to take a good snapshot for the target module. Some considerations include (a) avoidance of sun reflections, (b) avoidance of the shadow of the drone on the module as this may cause false alarms (especially with thermal cameras), (c) the dynamic sun-tracking structure of the modules. More importantly, the entire inspection process is heavily reliant on the accuracy of the position of the drone, which can be improved by using multiple GPS receivers at the drone and by using a Real-Time Kinematic (RTK) positioning which is orders of magnitude more precise. Positioning will be discussed in more details in section III-F.

\section{WAYPOINT PLANNING}

\section{A. System Model}

In a typical UAV based inspection system for large-scale solar farms, it is necessary to hover the UAV over (or close to) solar modules and control the gimbal mounted camera that takes snapshots to accurately identify the defects [11]. This imposes the requirement of accurate 3D positioning.

Our system model is illustrated in Fig. 3. Given that the waypoints have been planned and loaded, the UAV equipped with one or multiple sensors vertically takes-off from a defined position and lands at the same spot carrying out its mission. Though it can be fully autonomous, a supervising pilot is presently available to assist in the mission and ensure the safety of the solar farm structure and close-by personnel. Images acquired are uploaded to the processing center at the 


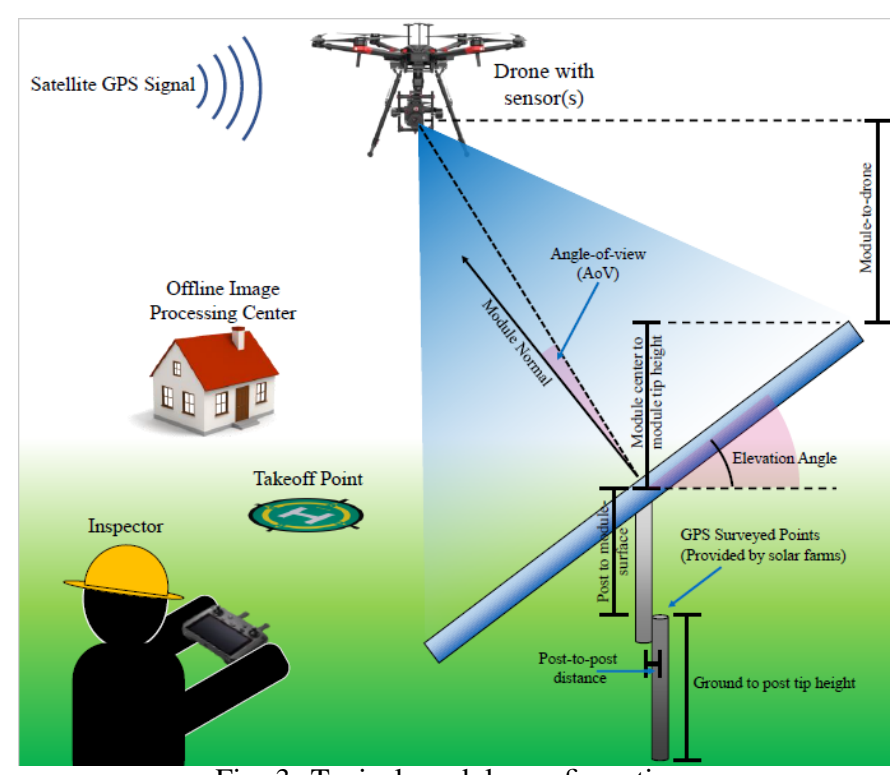

Fig. 3: Typical module configuration.

end of the flight. Generating the waypoints with the right drone and camera parameters from the surveyed GPS locations of the solar modules is a multivariate function depending on solar module structure, dimensions and tracking configuration, sampling policy, modules' inspection order, imaging conditions, and drone positioning. We use litchi [16] to interface waypoint generation to the UAV flightplan and the waypoints are defined in a compatible format ${ }^{1}$

$$
w_{k}=\left[\begin{array}{llllll}
x_{k} & y_{k} & z_{k} & \phi_{k}^{1} & \phi_{k}^{2} & \phi_{k}^{3}
\end{array}\right],
$$

where $x_{k}, y_{k}$, and $z_{k}$ are the longitude (decimal degrees), latitude (decimal degrees), and altitude (meters), while $\phi_{k}^{1}, \phi_{k}^{2}$ and $\phi_{k}^{3}$ are the roll, pitch, and yaw angles (all in degrees) of the camera orientation. These parameters are illustrated in Fig. 4. For the inspection task of solar modules, The roll $\phi_{k}^{1}$ of the camera is set to 0 without loss of generality. The pitch angle $\phi_{k}^{2}$ is defined in the interval $[-90,0]$ : When $\phi_{k}^{2}=0$, the camera is parallel to the horizon, and when $\phi_{k}^{2}=-90$, the camera is looking downwards (normal to the ground). The recommended $\phi_{k}^{2}$ value is $5^{\circ}$ away from the module's surface normal to avoid sun reflections (a function of the elevation angle that, in turn, is a function of the time of the day and the geographical location). The yaw $\phi_{k}^{3}$ is chosen parallel to the module's $y$ axis, that is, lining the camera up in the longitudinal axis of the module. In practice, the yaw command is often used as a set point to the orientation of the quad, rather than a set point to the gimbal on which the camera is mounted. The longitude and latitude parameters are obtained from surveyed data prior to the mission. A correction is added to account for the installation terrain map, the support structure of the module and the module's configuration and elevation angle. A second offset is added based on the time of day that the image will be taken to account for the expected orientation of the panel and locate the UAV in the desired position. The orientation of the panel can be computed from the time of the

\footnotetext{
${ }^{1}$ We skip action parameters such as taking images as they are implicit to the inspection process.
}

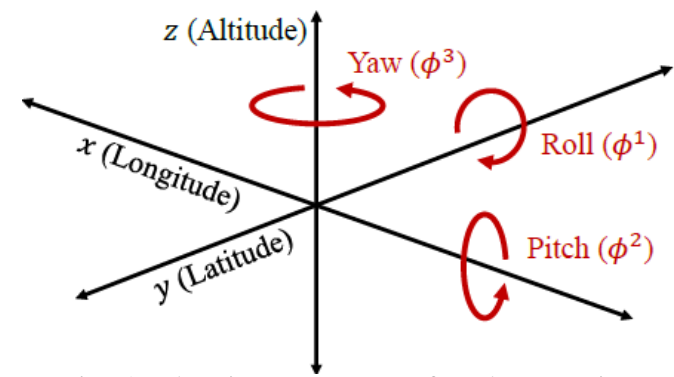

Fig. 4: The six parameters of each waypoint.

day. Should the tracking control of the panel be faulty, the poor orientation of the panel is obvious from the image and the image processing algorithm will note the panel as faulty.

\section{B. Solar Module Configurations}

Generating the exact waypoints needed for inspecting solar modules is a challenging task. There are multiple modulerelated parameters involved in the computation of the waypoints. For example, the sun-tracking nature of solar modules makes it difficult to keep the same angle of view (AoV) for all inspected modules and image and to keep $\phi_{k}^{2}$ away from the direct sun reflection for all target modules.

As illustrated in Fig. 3, the module's elevation angle is a key parameter to determine the flight altitude that will give an almost identical image. That is, the inspection altitude is a function of the elevation angle ${ }^{2}$, which also determines the highest tip of the module. Analytically, for a module of length $L$ and width $W$, the change in the altitude, $\Delta_{\text {alt }}$ in meters, with respect to the surveyed coordinates due to a non-zero elevation angle, $\theta$, is given by

$$
\Delta_{\text {alt }}=\frac{W}{2} \sin (\theta)
$$

\section{Modules Sampling and Inspection Confidence}

Solar farms typically have hundreds of thousands of installed modules. Even with a UAV, aerial inspection can take a significant amount of time, though it is still way faster than manual inspection. As such, and assuming that modules are independent from each other, a reasonable action is to randomly inspect a sample set of modules of size $\mathcal{K}$ to represent the whole solar farm population of size $\mathcal{N}$, while being confident that the $\mathcal{K}$ modules represent the $\mathcal{N}$ modules with some confidence level $\mathcal{C} \in[0,1]$ and a given error margin probability $\varepsilon$. To this end, $\mathcal{K}$ is calculated as [17]

$$
\mathcal{K}=\left\lceil\frac{\xi}{1+\xi / \mathcal{N}}\right\rceil
$$

where

$$
\xi=\frac{z^{2} p(1-p)}{\varepsilon^{2}},
$$

and $p \in[0,1]$ is a proportionality constant, and $z$ is the $\mathrm{z}$-score calculated as

$$
z=\sqrt{2} \mathcal{Q}(1-\mathcal{C}) ;
$$

where $\mathcal{Q}(-)$ is the inverse complementary error function. To

\footnotetext{
${ }^{2}$ Extension to dual-axis trackers is straightforward.
} 


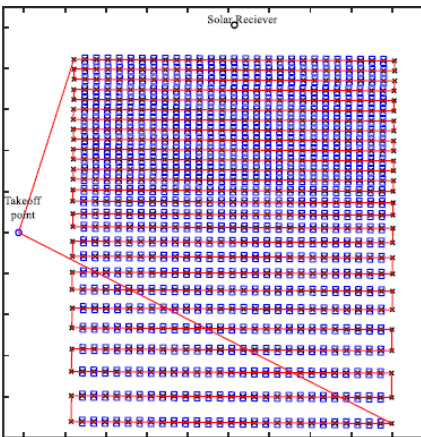

(a)

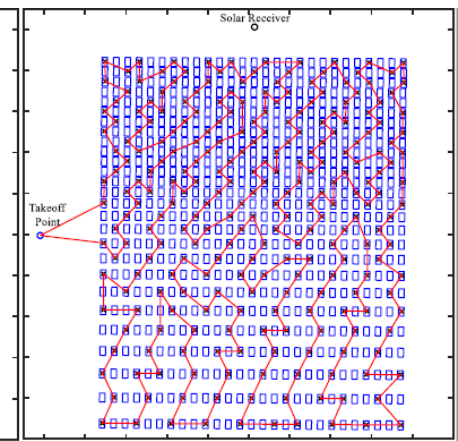

(b)
Fig. 5: (a) Full-search and (b) TSO waypoints planning for an example heliostat field.

illustrate the usefulness of this sampling approach, imagine a solar farm with $\mathcal{N}=10,000$ modules, and we require a confidence level $\mathcal{C}=0.95$ and an error probability $\varepsilon=0.05$, then one needs to randomly inspect $\mathcal{K}=370$ modules only.

\section{Efficient Waypoint Planning}

As UAVs typically fly well-above solar modules, which are normally installed in flat and open areas, some research work (e.g. [11]) considered flight planning without accounting for vertical movement (fixed altitude). As such, waypoint generation is regarded as 2D and obstacle-free [11]. However, this planning is not suitable for inspection tasks since highresolution images require the vehicle to approach closely to within $4 \sim 5$ meters from the surface of the module.

While the sampling approach mentioned earlier reduces the number of modules to be inspected, the selection of the $\mathcal{K}$ modules is still random. As such, the modules have to be ordered to be inspected such that the flight time is minimal while covering the maximum number of modules and using the same set of batteries. Specifically, given a set of waypoints $w_{1}, w_{2}, \ldots, w_{\mathcal{K}}$, it is necessary to find a parametric path in $\mathbb{R}^{3}$ that passes through all $\mathcal{K}$ waypoints in the shortest and optimal order. Two path planning methods are considered: (a) full-search and (b) travelling-salesman path planning.

1) Full-Search: Full-search is conducted when an exact detailed report is required for each module in the solar farm. It is typically useful for small solar farms or to monitor the installation of solar farms as it progresses. In such cases, the full search is conducted across each row of modules. Switching from one row to the next is done in a zigzag way. Fig. 5 (a) illustrates a typical path planning for full-search inspection of an example heliostat field.

2) Travelling Salesman Search: A sample of modules is selected as a representation of the solar farm using a suitable random algorithm. Base waypoints are generated for each module. For each pair of waypoints, the distance between the waypoints can be easily computed, and the resulting minimum time path to visit all modules in the samples is well defined. This problem can be solved using the travelling salesman search optimization (TSO) [18]. The travelling salesman problem finds the cheapest solution (where the cost, in this case, is the travel distance) and is not influenced by the inspection order. Starting with the take-off point and ending in the same

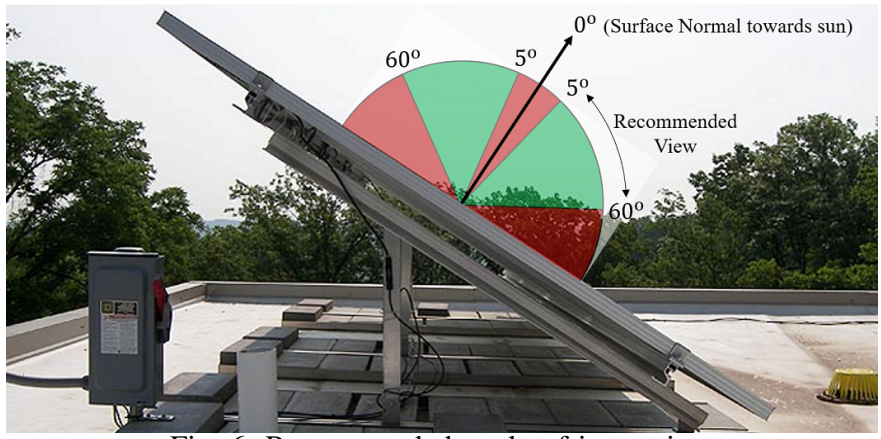

Fig. 6: Recommended angle of inspection.

place, the optimizer returns the order in which modules should be inspected. Finally, the precise set of waypoints is computed based on adding the offsets (dictated as mentioned earlier by the panel angle, sun position, clearance above the panel, etc) to the base waypoints in order and computing the time-ofday that each waypoint will be visited, based on an assumed takeoff time. The final verification is to ensure that the flight time does not exceed the battery capacity of the UAV. If the path is too long then the sample number is reduced and the algorithm recomputed. This is unlike standard robotic planning algorithms that are interested in a path between two points rather than a path that visits all points. Fig. 5 (b) illustrates a TSO waypoint plan for a random set of heliostats.

\section{E. Imaging and dataset generation}

Cameras/sensors play a key role in the inspection process. The inspection capability and the required flight altitude to capture detailed module images are correlated [13, 14]. One also needs to keep in mind avoiding normal-to-surface viewing as sun reflections can saturate images and hence negatively affect the inspection. The recommendation by FLIR [19] is to stay within $5^{\circ} \sim 60^{\circ}$ with respect to the module surface normal for thermal images as in Fig. 6.

If camera resolution is a limitation and an image of the entire module or mirror does not yield sufficient level of detail, then multiple images of the same panel may be taken. In this case, a waypoint is "burst" into four, where each new waypoint is centered at the middle of the four quadrants of the module.

\section{F. Positioning and RTK}

Field experiments show that there can be significant positioning errors from the drone's GPS receiver(s) or gimbal. The authors in [20] characterized this position noise as a white Gaussian noise and assumed the position uncertainty at each waypoint to be independent from the previous ones. That is,

$$
\widehat{w}_{k} \sim \mathcal{G}(\mu, \sigma),
$$

where $\mathcal{G}(\mu, \sigma)$ is a Gaussian process with mean $\mu$ and variance $\sigma$, which are respectively calculated as $\mu=\mathcal{E}\left(\widehat{w}_{k}\right)$ and $\sigma=\mathcal{E}\left[\left(\widehat{w}_{k}-\mu\right)\left(\widehat{w}_{k}-\mu\right)^{\top}\right][20]$. In (6), $\mathcal{E}(-)$ is the expectation operator. Drift can be as severe as a few meters. Based on our field observations, the drift can be also quasistatic. While having multiple GPS receivers on the drone can slightly alleviate this problem, even minor drift, of $20 \mathrm{~cm}$ or 


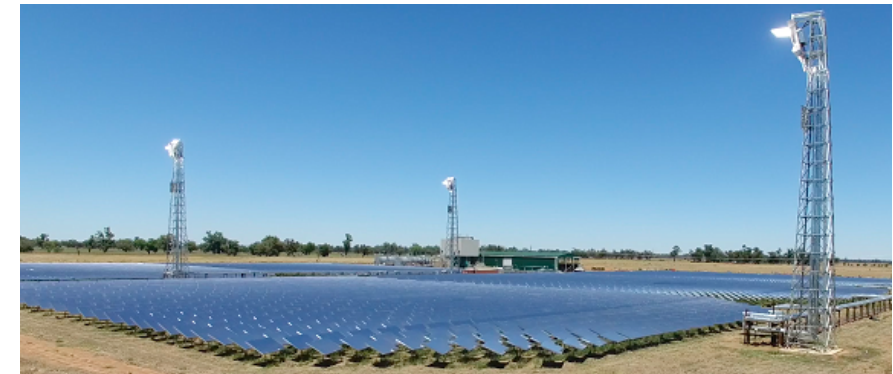

Fig. 7: Experiments location at Vast Solar, New South Wales.

more, leads to unacceptable compromise of the image quality. The solution to drift is two-fold.

1) Drift estimation and correction: Before taking off, one can measure the amount of drift, e.g., by comparing a presurveyed GPS reading vs. the drone's current GPS reading at the same landmark (such as the takeoff point). Then, all waypoints can be shifted to account for this offset. This approach doesn't need any extra hardware but since GPS drift is highly correlated to the satellite configuration that changes regularly, these corrections are not stable in time.

2) RTK: Integration of Real-time Kinematics (RTK) system in the Global Navigation Satellite System (GNSS) information [21]. RTK uses a fixed receiver that sends out position correction information to the drone based on a well-surveyed base station. It performs a similar correction to GPS offset mentioned above but is continuously (usually every 1 second) updated during the flight instead of one time before takeoff. The accuracy of RTK is incomparable with that of GPS receivers. RTK has been successfully used in UAV system to geo-reference defective cells in [22].

\section{EXPERIMENTS AND APPLICATIONS}

\section{A. Hardware for experiments}

The choice of different UAV properties and relative sensors is essential in order to make the most appropriate inspection platform. To test our waypoints generation procedure in real flights, the DJI Matrice 600 [23], a powerful hex-copter that is particularly designed for applications such as professional photography and industrial inspections, was used.

The hex-copter was equipped with a DJI Zenmuse X5, a high-resolution camera that is suitable for inspection applications with its 16-megapixel images.

\section{B. Flight Missions and Dataset Collection}

With the assistance of our industrial partners in Australia, thousands of images were collected from several flight missions. However, unlike the first data collection for PV images from the Williamsdale solar farm where waypoints planning was done manually, at the Vast Solar Pilot Plant at Jemalong, NSW, we utilized the provided surveyed heliostat locations and used the presented waypoints generation algorithm to autonomously control the drone and the camera to capture images. The experimental field is shown in Fig. 7. As the field sampling feature was not part of the algorithm at that time, dataset collection during this first mission was done using full search. The planned flight for this mission is shown in

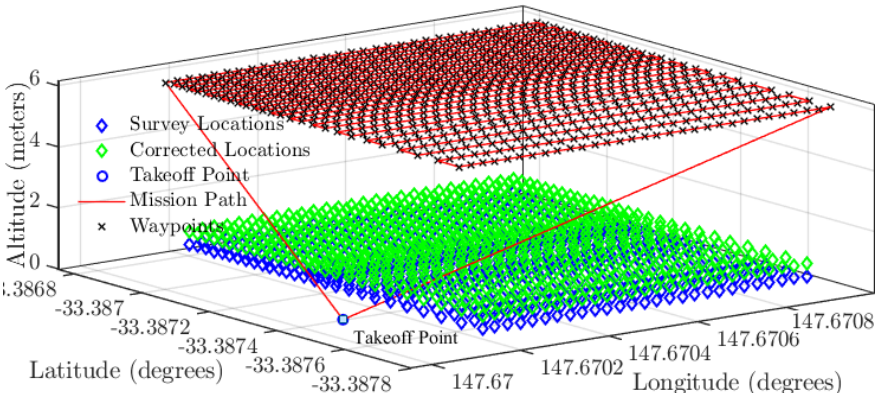

Fig. 8: 3D mission plan for Vast Solar.

Fig. 8. The proposed "burst" imaging was also successfully demonstrated in this mission.

During these test flights, the issue of GPS drifting was first noted to be a critical positioning challenge that hinders inspection accuracy, although pre-flight drift correction was useful in alleviating it. The need for RTK based positioning became evident.

To test the accuracy of RTK systems, we conducted a simple experiment where the UAV was positioned over the takeoff point without flying. Ideally, there should be zero change in the altitude and longitude data. To analyze the positioning error, location data were logged for around 15 minutes with the drone's standard GPS (no RTK), and in a second experiment with RTK integration. The data from these two experiments are shown in Fig. 9 and Table I summarizes the error statistics.

As indicated in Table I, the integration of RTK allows huge positioning gain over standard GPS, which renders it suitable for accurate positioning for inspection tasks. RTK will be tested in real flights along with the field sampling method and TSO waypoint planning to gather soiling data from our future visits.

\section{Application to PV Fault Inspection}

PV modules have several types of defects that degrades their ability to convert light into electricity. Some faults are visible to the naked eye and some are not. As such, inspecting for these faults requires multi-sensory (or multi-modal) data for every model. In [24], we tackled the problem of multimodal data registration for PV modules inspection by maximizing mutual information between the two modalities. This approach also accurately localizes each PV module with a sub-pixel accuracy. The reader is referred to [24] for more details.

\section{Conclusion}

In this research, we presented a UAV waypoints generation system that is specifically designed for aerial inspection of solar infrastructure. The system accounts for the physical structure and the dynamic nature of sun-tracking solar modules and generates waypoints with the right inspection configuration. Statistical sampling methods and TSO were used to generate a set of representative waypoints that are efficiently visited to study the status of solar farms within a userdefined confidence level and margin of error. Illustrative flights and preliminary PV and heliostats inspection results were discussed as the first step of more elaborative future research. In future work, we aim to include the terrain map as an input 


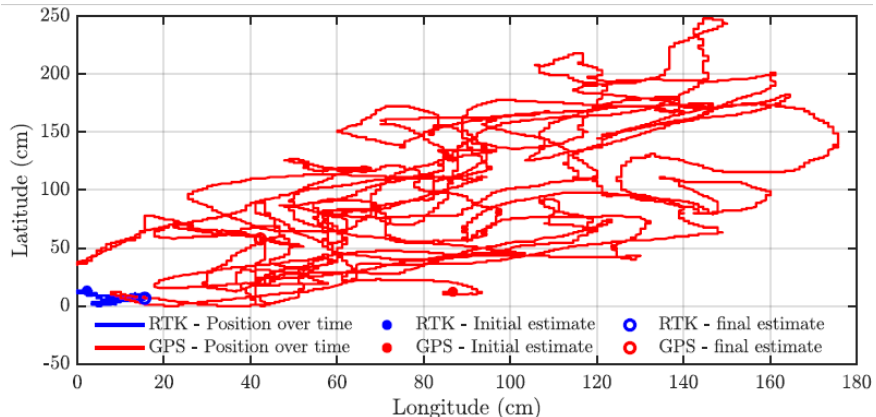

Fig. 9: RTK vs GPS position accuracy testing w.r.t. home location. UAV is stationary on the ground.

to our waypoints planning algorithm and study using multiple cooperative UAVs.

\section{ACKNOWLEDGEMENTS}

This research was supported by the Australian Renewable Energy Agency (ARENA), through Grant G00853 "A robotic vision system for rapid inspection and evaluation of solar plant infrastructure".

\section{REFERENCES}

[1] G. E. Tverberg, "Oil supply limits and the continuing financial crisis," Energy, vol. 37, no. 1, pp. 27 - 34, 2012. 7th Biennial Int. Workshop on Advances in Energy Studies.

[2] Australian Energy Resources Assessment, "Australian energy resources assessment," 2018.

[3] G. Spagnuolo, W. Xiao, and C. Cecati, "Monitoring, diagnosis, prognosis, and techniques for increasing the lifetime/reliability of photovoltaic systems," IEEE Trans. Ind. Electron., vol. 62, pp. 7226-7227, Nov 2015.

[4] S. Djordjevic, D. Parlevliet, and P. Jennings, "Detectable faults on recently installed solar modules in western australia," Renewable Energy, vol. 67, pp. 215 - 221, 2014. Renewable Energy for Sustainable Development and Decarbonisation.

[5] Agilent Technologies, "Solutions for solar cell and module testing," 2018.

[6] A. Dolara, G. C. Lazaroiu, S. Leva, G. Manzolini, and L. Votta, "Snail trails and cell microcrack impact on pv module maximum power and energy production," IEEE J. Photovolt., vol. 6, pp. 1269-1277, Sept 2016.

[7] Ã. Netland, G. Jenssen, H. M. Schade, and A. Skavhaug, "An experiment on the effectiveness of remote, robotic inspection compared to manned," in 2013 IEEE Int. Conf. Syst., Man, Cybern., pp. 2310-2315, Oct 2013.

[8] J. E. See, "Visual inspection: a review of the literature," Sandia Report SAND2012-8590, Sandia National Laboratories, Albuquerque, New Mexico, 2012.

[9] F. Grimaccia, S. Leva, A. Dolara, and M. Aghaei, "Survey on pv modulesâ common faults after an o\&m flight extensive campaign over different plants in italy," IEEE J. Photovolt., vol. 7, pp. 810-816, May 2017.
[10] Z. He and L. Zhao, "The comparison of four uav path planning algorithms based on geometry search algorithm," in 2017 9th Int. Conf. on Intelligent HumanMach. Systems and Cybernetics (IHMSC), vol. 2, pp. 3336, Aug 2017.

[11] X. Luo, X. Li, Q. Yang, F. Wu, D. Zhang, W. Yan, and Z. Xi, "Optimal path planning for uav based inspection system of large-scale photovoltaic farm," in 2017 Chinese Automation Congress (CAC), pp. 4495-4500, Oct 2017.

[12] I. A. Musliman, A. A. Rahman, V. Coors, et al., "Implementing 3d network analysis in 3d-gis,"

[13] S. Leva, M. Aghaei, and F. Grimaccia, "Pv power plant inspection by uas: Correlation between altitude and detection of defects on pv modules," in 2015 IEEE 15th Int. Conf. Environ. Elect. Eng., pp. 1921-1926, June 2015.

[14] M. Dalsass, S. Deitsch, P. Luchscheider, F. Gallwitz, and C. J. Brabec, "Performance of a module and defect detection algorithm for aerial infrared images as a function of the flying altitude," in 32nd European Photovoltaic Solar Energy Conf. and Exhibition, pp. 0613-0618, June 2016.

[15] Liang Yang, Juntong Qi, J. Xiao, and Xia Yong, "A literature review of uav $3 \mathrm{~d}$ path planning," in Proc. the 11th World Congress on Intelligent Control and Automation, pp. 2376-2381, June 2014.

[16] Litchi, "Litchi for dji mavic," 2019.

[17] J. L. Devore, Probability and Statistics for Engineering and the Sciences. Cengage learning, 2016.

[18] D. L. Applegate, R. E. Bixby, V. Chvatal, and W. J. Cook, The traveling salesman problem: a computational study. Princeton university press, 2006.

[19] FLIR, "FLIR systems." https://www.flir.com.au. Accessed: 2019-05-10.

[20] M. Kang, Y. Liu, Y. Ren, Y. Zhao, and Z. Zheng, "An empirical study on robustness of uav path planning algorithms considering position uncertainty," in 2017 12th Int. Conf. on Intelligent Systems and Knowledge Eng. (ISKE), pp. 1-6, Nov 2017.

[21] P. Addabbo, A. Angrisano, M. L. Bernardi, G. Gagliarde, A. Mennella, M. Nisi, and S. L. Ullo, "Uav system for photovoltaic plant inspection," IEEE Aerosp. Electron. Syst. Mag., vol. 33, pp. 58-67, August 2018.

[22] M. Nisi, F. Menichetti, B. Muhammad, R. Prasad, E. Cianca, A. Mennella, G. Gagliarde, and D. Marenchino, "Egnss high accuracy system improving photovoltaic plant maintenance using rpas integrated with low-cost rtk receiver," in Proc. Global Wireless Summit Conf., 2016.

[23] DJI, "Matrice 600." https://www.dji.com/au/matrice600. Accessed: 2019-05-10.

[24] E. Salahat, J. Coventry, A. Thomson, and R. Mahony, "Multi-modal image registration for robotic aerial inspection using mutual information," in 2018 Australasian Conf. on Robotics and Automation, 2018. 
TABLE I: Positioning error comparison with and without RTK.

\begin{tabular}{|l|c|c|c|}
\hline \multicolumn{1}{|c|}{ Reading } & RTK (cm) & GPS (cm) & Gain \\
\hline \hline Longitude (avg) & 12.07 & 87.57 & 7.26 \\
\hline Longitude (std) & 3.24 & 39.21 & 12.10 \\
\hline Latitude (avg) & 7.34 & 98.58 & 13.43 \\
\hline Latitude (std) & 1.89 & 54.07 & 28.61 \\
\hline
\end{tabular}

